\title{
Localized surface plasmon resonance in silver nanoparticles: Atomistic first-principles time-dependent density-functional theory calculations
}

\author{
M. Kuisma,${ }^{1,2, *}$ A. Sakko, ${ }^{3}$ T. P. Rossi, ${ }^{3}$ A. H. Larsen,${ }^{4}$ J. Enkovaara, ${ }^{5,3}$ L. Lehtovaara, ${ }^{6}$ and T. T. Rantala ${ }^{1}$ \\ ${ }^{1}$ Department of Physics, Tampere University of Technology, P.O. Box 692, FI-33101 Tampere, Finland \\ ${ }^{2}$ Department of Microtechnology and Nanoscience, MC2, Chalmers University of Technology, SE-41296 Göteborg, Sweden \\ ${ }^{3}$ COMP Centre of Excellence, Department of Applied Physics, Aalto University School of Science, FI-00076 AALTO, Finland \\ ${ }^{4}$ Nano-Bio Spectroscopy Group and European Theoretical Spectroscopy Facility (ETSF), Universidad del País Vasco UPV/EHU, \\ Avenida de Tolosa 72, E-20018 Donostia-San Sebastián, Spain \\ ${ }^{5}$ CSC-IT Center for Science Ltd., P.O. Box 405, FI-02101 Espoo, Finland \\ ${ }^{6}$ Department of Chemistry, Nanoscience Center, University of Jyväskylä, FI-40014 Jyväskylä, Finland
}

(Received 12 August 2014; revised manuscript received 4 March 2015; published 24 March 2015)

\begin{abstract}
We observe using ab initio methods that localized surface plasmon resonances in icosahedral silver nanoparticles enter the asymptotic region already between diameters of 1 and $2 \mathrm{~nm}$, converging close to the classical quasistatic limit around $3.4 \mathrm{eV}$. We base the observation on time-dependent density-functional theory simulations of the icosahedral silver clusters $\mathrm{Ag}_{55}(1.06 \mathrm{~nm}), \mathrm{Ag}_{147}(1.60 \mathrm{~nm}), \mathrm{Ag}_{309}(2.14 \mathrm{~nm})$, and $\mathrm{Ag}_{561}$ $(2.68 \mathrm{~nm})$. The simulation method combines the adiabatic GLLB-SC exchange-correlation functional with real time propagation in an atomic orbital basis set using the projector-augmented wave method. The method has been implemented for the electron structure code GPAW within the scope of this work. We obtain good agreement with experimental data and modeled results, including photoemission and plasmon resonance. Moreover, we can extrapolate the $a b$ initio results to the classical quasistatically modeled icosahedral clusters.
\end{abstract}

DOI: 10.1103/PhysRevB.91.115431

\section{INTRODUCTION}

Localized surface plasmon resonances (LSPRs) of silver nanoparticles (AgNPs) exhibit strong UV-visible absorption. The LSPRs can be tuned by fabrication techniques [1], or by functionalization [2], and they are sensitive to the nanoparticles' environment [3]. The sensitivity and tunability of AgNPs can be utilized in sensing [4], surface-enhanced spectroscopies [5], plasmon-enhanced chemistry [6], and photovoltaic applications [7]. Much of the wide interest in AgNPs originates from their role as building blocks of nanophotonic devices, such as optical nanoantennas [8]. The ability to predict the relation between their structure and operation is crucial for the applications. The optical characteristics of large noble-metal NPs $(>10 \mathrm{~nm})$ are well known, and their LSPRs can be simulated using classical electromagnetic theory. For example, large spherical AgNPs have a LSPR at $355 \mathrm{~nm}(3.5 \mathrm{eV})$, whereas icosahedral particles are slightly redshifted and have broader absorption arising from several LSPR modes that overlap closely in energy $[9,10]$. However, as the diameter of the NPs decreases, the LSPR blueshifts with the frequency being inversely proportional to the diameter [11] and, finally, the absorption spectrum changes to a typical cluster spectrum characterized by several individual transitions between quantized energy levels $[12,13]$. For diameters smaller than $10 \mathrm{~nm}$, the sensitivity of the LSPRs to the shape and surroundings of the AgNP becomes important, as is reflected in the difficulty of interpretation of experiments.

Previous theoretical studies on the LSPRs in AgNPs are limited to quantum mechanical calculations of small clusters [14] and jellium models [15], or to classical electromagnetic theory

\footnotetext{
*mikael.kuisma@chalmers.se
}

PACS number(s): 73.20.Mf, 31.15.xr, 31.50.Df, 36.40.-c

for large NPs [9]. Between diameters of 1 and $5 \mathrm{~nm}$, the classical electromagnetic theory does not provide an adequate description of the possible quantum effects as it is scale invariant and therefore predicts no size dependence. Jellium models ignore — or at best approximate [16] - the effects of $d$ electrons and atomic structure which are crucial to the proper description of AgNPs. Ab initio methods are limited to small clusters: Time-dependent [17] (TD) density-functional theory $[18,19]$ (DFT) has been used to model $\mathrm{Ag}_{55}$ and $\mathrm{Ag}_{147}$ [14], as well as nanoshells up to $\mathrm{Ag}_{272}$ [20].

Typically in such studies one uses the adiabatic local density approximation (ALDA) or the adiabatic generalized gradient approximation (AGGA) as exchange-correlation (XC) functionals, even though the LDA and GGA are known to predict a too-high-lying $d$-electron band and therefore to severely overestimate the $d$-band screening [21]. This results in decreased oscillator strength and lowered plasmonic frequency compared to experimental results. Both experimental [22] and theoretical [23] works have confirmed that the position of the $d$ band strongly influences the plasmonic properties. Quantitative theory must be based on a more accurate description of the $d$ band.

A recent experimental study of Scholl et al. found quantum effects influencing the optical properties of AgNPs with diameters as large as $10 \mathrm{~nm}$ [24]. In particular, their electron-energyloss spectroscopic (EELS) measurements on NPs showed a significant $(0.5 \mathrm{eV})$ blueshift of the LSPR when the diameter decreased from $7 \mathrm{~nm}$ to $2 \mathrm{~nm}$. This disagrees with previous experimental results for free-standing clusters [11,25], and Haberland has suggested that the blueshift is not due to the quantum effects but due either to the interaction of the LSPR with the substrate or the residual ligand molecules [26]. This controversy exemplifies that without tools that can simulate the optical properties of NPs from molecular size up to the classical limit, it is difficult to separate the quantum effects from other factors. 
In this work, we present an ab initio theoretical analysis of freestanding AgNPs up to a diameter of $2 \mathrm{~nm}$, and show that it is unlikely that the results of Scholl et al. correspond to freestanding AgNPs. Using atomistic first-principles calculations in the TDDFT framework, we are able to obtain macroscopic LSPRs already at a diameter of $2 \mathrm{~nm}$. Our calculations show that the resonance shifts only by $0.2 \mathrm{eV}$ above that. Our results agree with the experimental cluster data for both the smallest and the largest structures. Concurrently with explaining the experimental findings, we show that accurate treatment of interband ( $d$-electron) excitations is crucial for a reliable description of AgNP plasmonics. Therefore, we recommend the adiabatic Gritsenko-van Leeuwen-van Lenthe-Baerends-solid-correlation potential (GLLB-SC) [27] for approximating the exchange and correlation effects for the optical properties of noble metal NPs. The potential is a modification of the GLLB-potential [28] to be better suited for solids and surfaces and with added correlation.

In Sec. II we describe the details of our implementation of linear combinations of atomic orbitals with time-dependent density-functional theory (LCAO TDDFT), and elaborate the relevance of the GLLBSC potential for the proper description of plasmonics. In Sec. III we give basic background information about the quantum mechanical and the electrodynamical models. In Sec. IV we analyze the results obtained, and compare them to experimental EELS and photoemission data. In Sec. V we carefully benchmark the accuracy of our method. In Sec. VI we summarize the results and discuss the relevance of a proper Kohn-Sham eigenvalue description for accurate absorption spectra in AgNPs.

\section{METHODS}

The main computational challenges in simulating the photoabsorption spectrum of nanoplasmonic structures using TDDFT are (1) the quality of the XC functional, especially for the description of the silver $d$ band, (2) the numerical discretization scheme for the wave functions and the density, which must be flexible enough to describe the LSPRs, and (3) the method for optical properties, which must be fast, able to be parallelized, and scale well with respect to system size. Each of the challenges will be addressed in the following sections.

\section{A. Time-dependent density-functional theory}

Time-dependent density-functional theory is a wellestablished tool for calculating electronic excitations. As in DFT, the most crucial aspect of TDDFT is the exchangecorrelation potential, which is time dependent in this case. The time-dependent Kohn-Sham (KS) equations for the electronic orbitals $\Psi_{i}$ are

$$
\left(-i \partial_{t}-\frac{1}{2} \nabla^{2}+v_{\mathrm{KS}}[n(\mathbf{r}, t)](\mathbf{r}, t)\right) \Psi_{i}(\mathbf{r}, t)=0,
$$

where $v_{\mathrm{KS}}$ is the Kohn-Sham potential, the time-dependent density is given by

$$
n(\mathbf{r}, t)=\sum_{i} f_{i}\left|\Psi_{i}(\mathbf{r}, t)\right|^{2},
$$

and $f_{i}$ are the occupation numbers of the orbitals.
In the general formalism, the exchange-correlation part of the Kohn-Sham potential $v_{\mathrm{XC}}$ depends causally on all previous densities. In a practical and widely used adiabatic approximation, the potential depends only on the instantaneous density. We will use this approximation also in the case of the GLLBSC potential, with one further modification, as discussed in the next section.

\section{B. Adiabatic GLLBSC potential}

Adiabatic (semi)local-density approximations, such as the ALDA and the AGGA, are applicable for nearly-free-electron metals, but for noble metals the situation is different because they overestimate the polarizability of $d$ electrons. This is due to their Kohn-Sham spectrum, since they predict a too delocalized $d$ band in addition to its being too shallow [21,29]. To overcome this problem we employ the adiabatic GLLBSC potential $[27,28]$ that includes the exchange-hole and correlation potential of the Perdew-Burke-Ernzerhof functional for solids and surfaces (PBEsol) [30], and is additionally supplemented by a computationally efficient approximation of the hole response part (see, e.g., Ref. [31]) of the exactexchange optimized effective potential [32].

The GLLBSC functional introduces an orbital-energydependent localization of the exchange hole which reduces self-interaction and yields better asymptotic behavior than the LDA or GGA [27]. So far the GLLBSC functional has been mostly applied for predicting semiconductor band gaps [27,33], but recently Yan et al. showed that it also yields good results for Ag surface plasmons because of the improved $d$-band description [21]. We employ this finding, but extend it further by applying the GLLBSC method also for the dynamic response (in Ref. [21], the GLLBSC functional was used only for the ground state whereas the linear response calculation employed the ALDA).

We obtain an adiabatic GLLBSC approximation by replacing the time-dependent response coefficients $w_{i}(t)$ with their time-independent ground-state values $w_{i}(t=0)=$ $K_{g} \sqrt{\epsilon_{f}-\epsilon_{i}}$ in the GLLBSC potential [see Eqs. (16) and (22) of Ref. [27]]:

$$
v_{\mathrm{GLLBSC}}(\mathbf{r})=v_{\text {xhole }}(\mathbf{r})+\sum_{i} w_{i}(t=0) \frac{\left|\psi_{i}(\mathbf{r})\right|^{2}}{n(\mathbf{r})}+v_{c}(\mathbf{r}),
$$

where $v_{\text {xhole }}(\mathbf{r})$ is the Coulomb potential due to the exchange hole obtained from the exchange hole of PBEsol evaluated at the instantaneous density, $v_{c}(\mathbf{r})$ is the semilocal PBEsol correlation potential, and the remaining term is an approximation to the response of the Coulomb potential of the exchange-correlation hole to density perturbations. We choose $w_{i}(t)=w_{i}(t=0)$, since it is the simplest obtainable approximation and computationally attractive. It is plausible that this approximation is accurate in our simulations because we apply a small perturbation which will not significantly change the density, and thus not induce large oscillations of $w_{i}(t)$. In addition, our preliminary adiabatic time-dependent Krieger-Li Iafrate (TDKLI) [34] calculations indicate that the effect of $w_{i}(t)$ compared to $w_{i}(t=0)$ in the systems considered here is negligible in the linear response regime. 
It is difficult to estimate the effect of this approximation, or the effect of nonadiabatic exchange-correlation effects exactly. However, there is much evidence from historical work that already the random phase approximation (pure Coulomb kernel) without any XC kernel is sufficient to describe plasmonics [35]. Therefore, the adiabatic GLLBSC approximation should be a sufficient description for AgNP plasmonics.

\section{Real time propagation with basis sets}

The wave functions are represented as linear combinations of atomic orbitals together with the projector-augmented wave method [36] (PAW) as implemented in the GPAW package $[37,38]$. The smooth pseudo-wave-functions are written as a linear combination

$$
\tilde{\Psi}_{i}(\mathbf{r}, t)=\sum_{\mu} C_{\mu i}(t) \tilde{\phi}_{\mu}\left(\mathbf{r}-\mathbf{R}^{\mu}\right)
$$

of atom-centered orbitals $\tilde{\phi}_{\mu}\left(\mathbf{r}-\mathbf{R}^{\mu}\right)$ with expansion coefficients $C_{\mu i}(t)$. The PAW projection operator [36] $\widehat{\mathcal{T}}$ can be used to reconstruct the all-electron functions as $\Psi_{i}(\mathbf{r}, t)=$ $\widehat{\mathcal{T}} \tilde{\Psi}_{i}(\mathbf{r}, t)$. The PAW form of the time-dependent Kohn-Sham equations (1) is

$$
\left[\widehat{\mathcal{T}}^{\dagger}\left(-i \frac{\partial}{\partial t}\right) \widehat{\mathcal{T}}+\widehat{\mathcal{T}}^{\dagger} \widehat{H}_{\mathrm{KS}}(t) \widehat{\mathcal{T}}\right] \tilde{\Psi}_{i}(\mathbf{r}, t)=0,
$$

where $\widehat{H}_{\mathrm{KS}}(t)$ is the Kohn-Sham Hamiltonian for noninteracting electrons.

Substituting Eq. (4) into Eq. (5) and multiplying with $\int d \mathbf{r} \tilde{\phi}_{\mu}(\mathbf{r})$ from the left, the equation can be cast into a matrix form

$$
i \mathbf{S} \frac{d \mathbf{C}(t)}{d t}=\mathbf{H}(t) \mathbf{C}(t),
$$

with the overlap matrix $S_{\mu \nu}=\left\langle\tilde{\phi}_{\mu}\left|\widehat{\mathcal{T}}^{\dagger} \widehat{\mathcal{T}}\right| \tilde{\phi}_{\nu}\right\rangle$ and the Hamiltonian matrix $H_{\mu \nu}(t)=\left\langle\tilde{\phi}_{\mu}\left|\widehat{T}^{\dagger} \widehat{H}_{\mathrm{KS}}(t) \widehat{T}\right| \tilde{\phi}_{\nu}\right\rangle . \mathbf{C}(t)$ is the matrix of LCAO expansion coefficients $\left\{C_{\mu i}(t)\right\}$ defined in Eq. (4). The overlaps $S_{\mu \nu}$ and the projection operator $\widehat{\mathcal{T}}$ are constant because the nuclei are assumed to be stationary.

In this approach, the time-dependent density and potential are expressed on a uniform grid, and the matrix elements of the potential are evaluated on this grid [38]. The smoothness of these quantities allows for a very coarse grid spacing, and the LCAO-PAW pseudo-wave-functions form a small, local, and efficient representation suitable for systems with hundreds of atoms [39].

We calculate the optical absorption spectrum of AgNPs using the time-propagation (TP) approach to TDDFT [40,41]. The greatest advantage of TP-TDDFT is the scaling of the computational requirements with respect to system size compared to other methods, such as Casida's approach [42]. Despite its better scaling, the large prefactor has so far limited the applicability of the TP-TDDFT approach.

Following the TP-TDDFT procedure for the optical response [43], we here excite the system by an instantaneous electric field $\mathbf{E}(\mathbf{r}, t)=E_{0} \hat{\mathbf{e}}_{\mathrm{tt}} \delta(t)$, where the field strength $E_{0}=$ 0.0001 a.u. is sufficiently small to avoid nonlinear effects, and the direction $\hat{\mathbf{e}}_{\mathrm{tt}}$ of the electric field is chosen to be from tip to tip, i.e., along the fivefold symmetry axis of the icosahedron. The optical absorption spectrum is obtained by
TABLE I. Performance of the LCAO-TDDFT code for time propagation of AgNPs for 1000 time steps of duration 10 as.

\begin{tabular}{lrrrrr}
\hline \hline System & Cores & Wall hours & CPU hours & Electrons & Basis functions \\
\hline $\mathrm{Ag}_{55}$ & 64 & 4.5 & 288 & 605 & 990 \\
$\mathrm{Ag}_{147}$ & 64 & 18.0 & 1152 & 1617 & 2646 \\
$\mathrm{Ag}_{309}$ & 256 & 28.5 & 7296 & 3399 & 5562 \\
$\mathrm{Ag}_{561}$ & 512 & 42.0 & 21504 & 6171 & 10098 \\
\hline \hline
\end{tabular}

Fourier-transforming the induced dipole moment along the excitation axis [40].

After the initial kick, the propagation is performed with a reliable and numerically stable semi-implicit Crank-Nicolson method. In brief, the method can be described as follows. In the prediction step, we solve

$$
\left(\mathbf{S}+i \frac{d t}{2} \mathbf{H}(t)\right) \mathbf{C}^{\prime}(t+\mathrm{d} t)=\left(\mathbf{S}-i \frac{d t}{2} \mathbf{H}(t)\right) \mathbf{C}(t),
$$

for $\mathbf{C}^{\prime}(t+d t)$, where $\mathbf{S}$ and $\mathbf{H}(t)$ are the basis set representations of the PAW overlap and Hamiltonian operator, respectively. The operations are parallel with matrices being distributed using the SCALAPACK [44] and BLACS [45] packages. After obtaining the initial approximation for the wave functions, the predict-correct method is applied. We then obtain an estimate for the Kohn-Sham Hamiltonian (including the XC potential) at the middle of the time step,

$$
\mathbf{H}(t+d t / 2) \approx\left[\mathbf{H}(t)+\mathbf{H}^{\prime}(t+d t)\right] / 2,
$$

where $\mathbf{H}^{\prime}(t+d t)$ is evaluated from $\mathbf{C}^{\prime}(t+d t)$, and then propagate the wave function to $t+d t$ in the correction step, which solves

$$
\begin{gathered}
\left(\mathbf{S}+i \frac{d t}{2} \mathbf{H}(t+d t / 2)\right) \mathbf{C}(t+d t) \\
=\left(\mathbf{S}-i \frac{d t}{2} \mathbf{H}(t+d t / 2)\right) \mathbf{C}(t),
\end{gathered}
$$

for $\mathbf{C}(t+d t)$. This results in $O\left(N^{3}\right)$ scaling with respect to the number of electrons in the system, to be compared to the GPAW's Casida implementation of $O\left(N^{5}\right)$ or the real-space time propagations $O\left(N^{2}\right)$. However, the constant factor in the grid propagation is so large that our scheme performs one to three orders of magnitude faster on systems of several thousand electrons. The timings for propagation are indicated in Table I.

\section{MODEL}

Both classical and quantum mechanical models are employed in this work. The quantum mechanical model is atomistic and $a b$ initio, relying only on DFT and TDDFT calculations. The plasmonic peak in our quantum mechanical model depends on both shape and size effects. The classical model is based on empirical dielectric functions and can model only shape effects. However, in the large-particle limit, the two methods should agree. Therefore, we can test the performance of our computational model also by extrapolating to the macroscopic Mie scattering limit.

In both models we consider icosahedral clusters. Charged $\mathrm{Ag}_{55}$ has been experimentally identified as icosahedral [46], 
and we choose to keep the icosahedral geometry to avoid shape effects even though the minimum-energy structure is expected to change for larger clusters [47]. The difference between icosahedron and sphere in the macroscopic limit is well understood $[9,10]$ and does not influence our conclusions. The atomic structure of icosahedral $\mathrm{Ag}_{55}$ used in our calculations includes a central atom with two icosahedral Mackay layers. We create clusters up to $\mathrm{Ag}_{561}$ by adding Mackay layers one by one, using a bond length of $3.0 \AA$. The ideal icosahedral clusters are then relaxed with the LDA functional. In these calculations the grid spacing is $0.2 \AA$, and the size of the cubic cell is chosen so that all atoms are at least $5.0 \AA$ away from the cell boundary. We use the default double- $\zeta$ polarized (DZP) basis set provided with GPAW for the geometry relaxations [38].

TDDFT simulations are performed for $30 \mathrm{fs}$ using time steps of 10 as. These calculations use a coarse grid spacing of $0.3 \AA$ and an expanded atomic basis set; we will further discuss these parameters in Sec. V. All spectra are calculated using a Gaussian broadening of $0.16 \mathrm{eV}$ FWHM.

In the following we consider classical electrodynamic approximations. First, the photoabsorption of a spherical NP of volume $V$ is given by the quasistatic limit of Mie theory:

$$
S(\omega)=\frac{3 V \omega}{2 \pi^{2}} \operatorname{Im}\left[\frac{\epsilon(\omega)-1}{\epsilon(\omega)+2}\right] .
$$

By using the experimentally determined permittivity $\epsilon(\omega)$ for silver presented in Ref. [48], Eq. (10) yields a strong LSPR at $3.5 \mathrm{eV}$. For more complicated shapes, such as icosahedra, one has to employ computational electrodynamics. In this work we use a quasistatic (QS) version [49] of the widely used finitedifference time-domain (FDTD) method, as implemented in GPAW [50]. As in photoabsorption calculations with TDDFT, in the QSFDTD method one perturbs the system by an external field and analyses the time-dependent dipole moment. The frequency-dependent dielectric permittivity of classical material is approximated using a set of Lorentzians. To obtain an accurate representation of the dielectric function of $\mathrm{Ag}$ especially near the LSPR, we start from the parametrization presented in Ref. [49] which uses nine Lorentzians, add one extra Lorentzian, and refit the dielectric function against the experimental data [48] with weight function $w(\omega)=$ $\exp \left[-(\omega / \mathrm{eV}-3.5)^{2}\right]$.

The QSFDTD calculations are performed using a regular grid of $96 \times 96 \times 96$ points. Since this method is size invariant, only a particle shape needs to be specified. We thus specify the shape as an icosahedron with a length of 40 points along its axis, securing adequate surrounding vacuum. The material is represented by a mask which assigns a value of either 1 (material) or 0 (vacuum) to each point. To ensure high numerical accuracy of the finite-difference operators, we smooth the edge of the icosahedron artificially over two to three grid points along the faces so that points along the faces are effectively a mixture of vacuum and silver.

\section{RESULTS}

Figure 1(a) shows the GLLB-SC TDDFT absorption spectra of icosahedral $\mathrm{Ag}_{55}, \mathrm{Ag}_{147}, \mathrm{Ag}_{309}$, and $\mathrm{Ag}_{561}$ clusters divided by the number of atoms in the system. For comparison, we present classical QSFDTD results for icosahedral (dashed

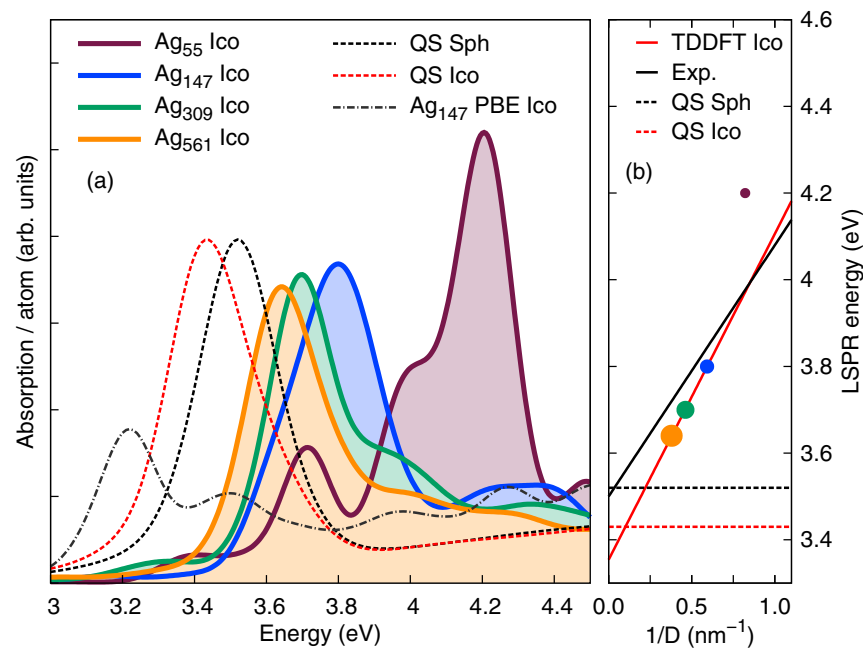

FIG. 1. (Color online) (a) Photoabsorption spectra for AgNPs normalized by the number of atoms. The spectra for icosahedral $\mathrm{Ag}_{55}, \mathrm{Ag}_{147}, \mathrm{Ag}_{309}$, and $\mathrm{Ag}_{561}$ are calculated with adiabatic GLLB-SC TDDFT. For comparison, the PBE-calculated spectrum of $\mathrm{Ag}_{147}$ is also shown. Classical QSFDTD spectra are shown for spherical (QS Sph) and icosahedral (QS Ico) AgNPs, calculated with the empirical dielectric function. These spectra are normalized using the empirical silver density and thus the peak strengths are directly comparable. (b) LSPR energy (circles) of icosahedral AgNPs as a function of inverse particle diameter calculated with GLLB-SC TDDFT. Solid black and red lines correspond to the experimental data for spherical NPs [11] and a linear fit to our results, respectively. Dashed horizontal lines represent classical limits (QSFDTD) for spherical (QS Sph) (3.52 eV) and icosahedral (QS Ico) (3.43 eV) NPs.

red) and spherical (dashed black) shapes. These correspond to the limit of large clusters as given by the quasistatic approximation. Figure 1(b) shows excitation energies of absorption peaks with respect to the inverse diameter of the cluster. For NPs larger than $\mathrm{Ag}_{55}$, the excitation energies of the most intense peak as a function of inverse diameter lie on a line (solid red) which extrapolates to $3.35 \mathrm{eV}$ in the large-particle limit, very close to the mesoscopic limit for icosahedral AgNPs at $3.43 \mathrm{eV}$ (dashed red) obtained from the QSFDTD calculation. The agreement of the quasistatic mesoscopic limit with the quantum mechanical asymptotic limit suggests that the quantum mechanical model correctly describes the shape effect. For comparison, a linear fit to experimental data (solid black) is shown for spherical AgNPs in an argon matrix [11] and also the mesoscopic limit for spherical NPs from the QSFDTD calculation (dashed black). The experimental values are shifted to the vacuum LSPR value of $3.5 \mathrm{eV}$ to account for the Ar matrix as suggested by Haberland [26]. The experimental and the simulated data show remarkable agreement, both in the asymptotic limit and in the size dispersion. The differences can be attributed to slightly different AgNP shape and structure. These observations suggest that the quantum mechanical model describes the finite-size effect in the AgNP plasmonics well.

In Fig. 1(a), in addition to the LSPR energy, the area of the plasmon peak per particle (oscillator strength) also agrees well with the classical electrodynamics simulation (dashed 
(a)

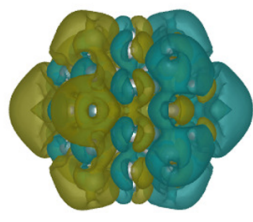

(c)

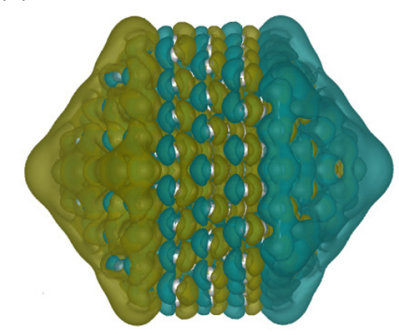

(b)

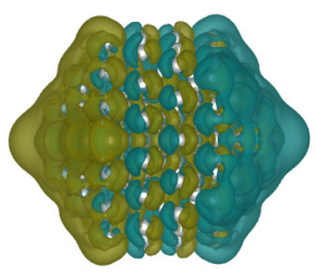

(d)

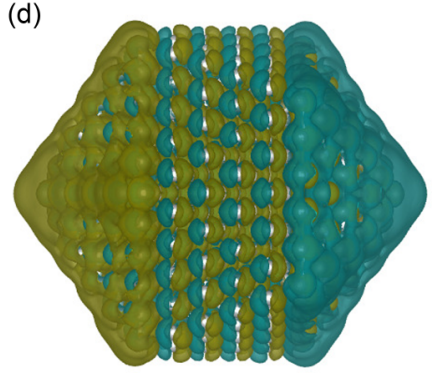

FIG. 2. (Color online) Calculated induced electron densities of LSPRs for (a) $\mathrm{Ag}_{55}$, (b) $\mathrm{Ag}_{147}$, (c) $\mathrm{Ag}_{309}$, and (d) $\mathrm{Ag}_{561}$. The Ag $s p$ band forms a localized surface plasmon on the surface of the cluster, whereas the $d$ electrons polarize in the opposite direction.

red). These observations strongly indicate that (i) the adiabatic GLLB-SC method provides realistic $d$-band screening in Ag nanostructures, and (ii) the macroscopic size range is reached for AgNPs of diameter $2 \mathrm{~nm}$. In Fig. 1, for comparison, we have included the spectrum of $\mathrm{Ag}_{147}$ calculated with the PBE functional [51]. It is important that the previous conclusions cannot be drawn from adiabatic PBE calculations because they underestimate the LSPR energy by $\sim 0.5 \mathrm{eV}$, and greatly underestimate the intensity, as seen on Fig. 1(a).

Previous works $[52,53]$ have demonstrated the importance of visual interpretation for characterizing the LSPRs in molecules and NPs. The induced electron densities of LSPRs in $A_{55}, A_{147}, A_{309}$, and $A_{5}$ are shown in Fig. 2. The exact quantity shown is the transition density at the plasmon frequency $\omega$ of each $\mathrm{AgNP}$, i.e., a sine transform

$$
\tilde{n}(\mathbf{r}, \omega)=\int_{0}^{\infty} d t[n(\mathbf{r}, t)-n(\mathbf{r}, 0)] e^{-\sigma^{2} t^{2} / 2} \sin \omega t
$$

of the charge-density fluctuation. The damping is given by $\sigma_{\text {FWHM }}=2 \sqrt{2 \ln 2} \sigma=0.16 \mathrm{eV}$. We observe that the Ag $s p$ band near the Fermi energy forms a localized surface plasmon mainly at the two opposing sides of the icosahedron, whereas $d$ electrons polarize in the opposite direction and thus create a counteracting screening field at the central region. This screening is overestimated by the PBE functional, causing the drop in plasmon energy and intensity. The figure corresponds to the classical picture of plasmons as a charge cloud oscillating between the opposite sides of the AgNP. The visual inspection thus supports our finding that a macroscopic plasmon forms in clusters of this size range.

Figure 3 shows the experimental photoemission data from two sources [54,55] on $\mathrm{Ag}_{55}$ compared to $s p$ - and $d$-band projected local densities of states of the quantum mechanical clusters. The $d$-band position of GLLB-SC matches well with the experimental data, as has also been observed earlier [21].

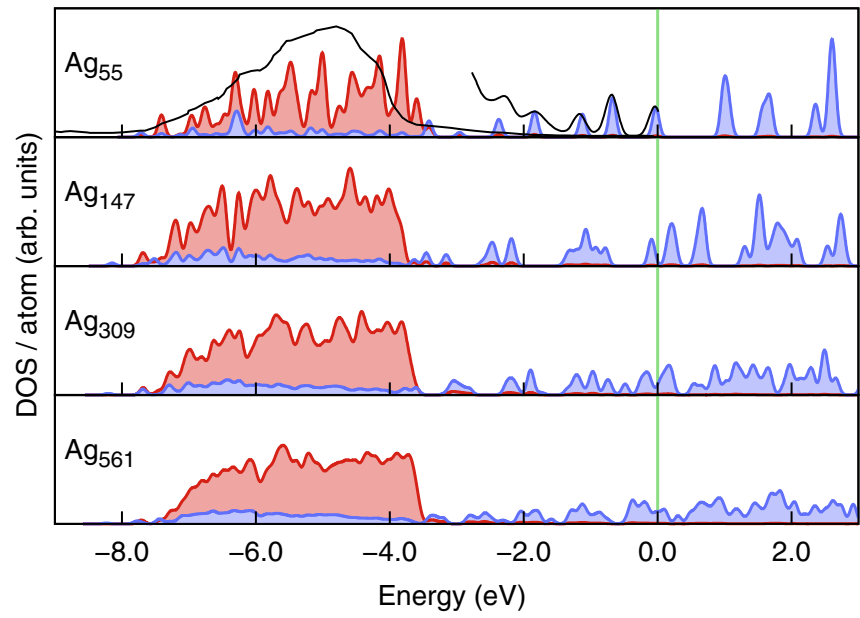

FIG. 3. (Color online) Density of states of icosahedral AgNPs calculated with the GLLB-SC functional, projected separately for the $d$ band (red) and $s p$ band (blue). The black curves are experimental data [54,55], shifted to align the Fermi levels. The charge state of $\mathrm{Ag}_{55}$ has an insignificant effect on the quantitative agreement.

In addition, the superatom shell description is in quantitative agreement with photoemission data [54].

\section{ACCURACY OF THE METHOD}

The PAW data set used to represent $\mathrm{Ag}$ includes the $5 \mathrm{~s}$ and $4 d$ orbitals as valence states, and is based on the default parameters of GPAW for the 11-electron Ag setup (e.g., the PBE Ag setup from GPAW setups v0.8.7929) but generated with the GLLB-SC functional.

In GPAW, one commonly uses a DZP numerical basis set to represent the wave functions [38]. This basis set includes the atomic Kohn-Sham orbital for each occupied valence state, one extra radial function for each atomic KS orbital generated using the standard "split-valence" scheme in GPAW, plus a polarization function which for transition metals is $p$ type. For the details of the construction of the basis sets, see Ref. [38]. This basis set is designed for ground-state calculations and would not be expected to (and indeed does not) accurately predict properties that depend on unoccupied states. To better represent the effect of the unoccupied $5 p$ orbitals, we replace the standard $p$-type polarization function with the actual Kohn-Sham orbital of the $5 p$ state plus its usual split-valence function.

Outside of this, we use the specific generation parameters [38] of $0.07 \mathrm{eV}$ confinement energy to localize the KS orbitals and a tail norm of 0.2 to define the range of the split-valence functions. These latter parameters we have optimized to provide an accurate density of states (DOS) in $\mathrm{Ag}_{55}$ as compared to an accurate real-space grid calculation, but this optimization has very little effect compared to the inclusion of the diffuse $5 p$ valence orbital. A comparison of DOSs is presented in Fig. 4. We observe that without the diffuse $5 p$ valence orbital the basis set is not able to reproduce the correct DOSs accurately, particularly for high energies. 


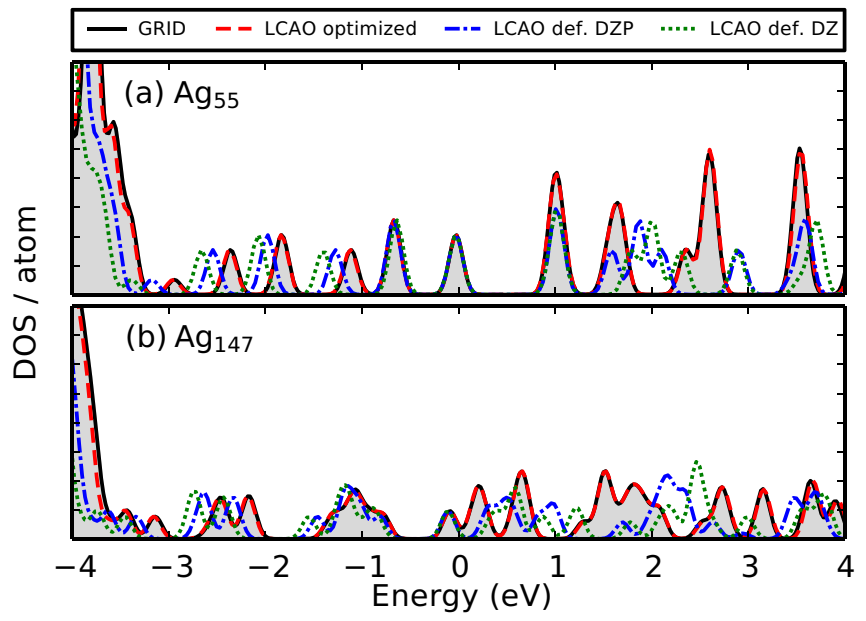

FIG. 4. (Color online) The density of states of (a) the $\mathrm{Ag}_{55}$ cluster and (b) the $\mathrm{Ag}_{147}$ cluster calculated with different LCAO basis sets as well as the grid mode.

Figure 5 presents the photoabsorption spectrum of the $\mathrm{Ag}_{147}$ cluster calculated with different basis sets and on a real-space grid. As in the DOS comparison, we note that the enhanced basis yields significantly better agreement with the grid mode than the default basis sets. In comparison to the real-space calculation, the enhanced LCAO basis reproduces the spectrum within $\sim 0.1 \mathrm{eV}$ and $\sim 5 \%$ accuracy for peak energy and intensity, respectively. This approach yields a transferable basis set that can be expected to describe both the DOS and the optical response of larger clusters with good accuracy. To obtain further improvement in accuracy, more elaborate approaches can be used to enhance the basis set [56].

To obtain good convergence with respect to the vacuum size, it is essential not to use zero boundary conditions for solving the Hartree potential of the Poisson equation. In the current work, we employ a multipole moment expansion [57] in order to obtain the correct boundary values of the Hartree potential. This allows us to scale down the required amount of vacuum from $15 \AA$ to $5 \AA$ and obtain significant speedup.

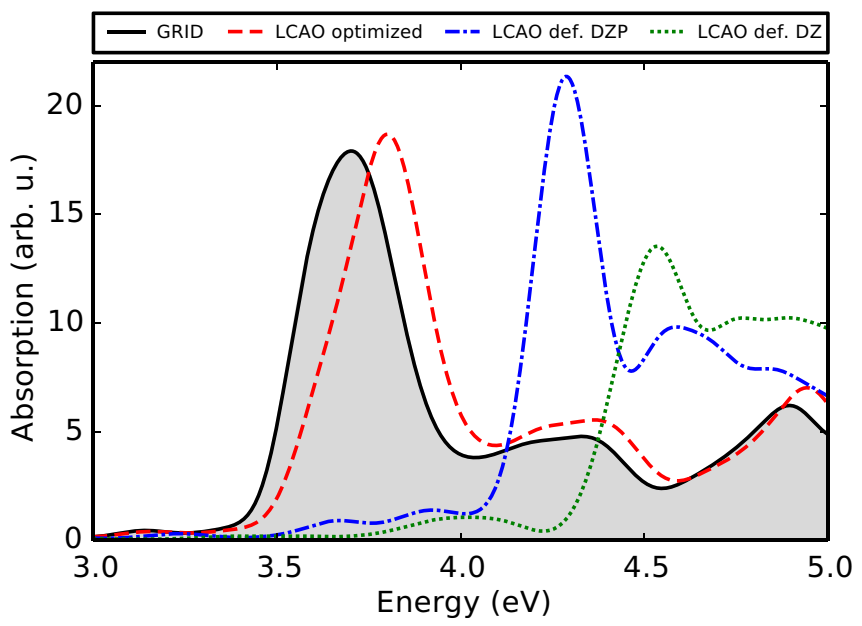

FIG. 5. (Color online) The photoabsorption spectrum of the $\mathrm{Ag}_{147}$ cluster calculated with different LCAO basis sets and the grid mode.
As indicated by Table I, our method achieves good parallel scaling in the weak sense, i.e., the computational time can be kept within reasonable limits by increasing the number of CPU cores as the system size increases.

\section{CONCLUSIONS}

We have established and carefully benchmarked a realtime propagation method using atomic basis sets to obtain accurate plasmonics as demonstrated here for icosahedral silver clusters. The implementation is part of the free opensource GPAW package. We have shown that the eigenvalue spectrum of the GLLB-SC potential matches the available experimental photoemission data for icosahedral silver clusters and that the method provides an accurate description of the plasmonic response in TDDFT calculations.

The observation that only the LSPR of $\mathrm{Ag}_{55}$ does not fit the asymptotic line in Fig. 1(b) suggests that the macroscopic regime is reached already at $\mathrm{Ag}_{147}$. However, comparison of the spectrum of $\mathrm{Ag}_{147}$ with the larger clusters shows that the shape of the LSPR peak deviates from that of the larger clusters. These quantum effects disappear for $\mathrm{Ag}_{309}$ and larger clusters. This threshold size for asymptotic LSPR behavior is remarkably small and agrees with experimental observations $[11,26]$ as well as with simulations of monolayerprotected Au clusters [53].

The impact of this study is threefold. First, we show using $a b$ initio simulations that the LSPR frequencies and intensities in icosahedral AgNPs enter an asymptotic region already around the diameter of $2 \mathrm{~nm}$. The optical response converges close to the classical limit of $3.43 \mathrm{eV}$ for icosahedral AgNPs. Our simulations are in good agreement with the experimental data, and the conclusion is further supported by visual examination and analysis of the DOSs. The presented results thus set a benchmark for the plasmonics of AgNPs, and explain the disparity between the recent EELS results and previous cluster experiments [24,26]. Second, the results show that the adiabatic GLLB-SC functional provides an accurate description of $d$-band screening in Ag nanostructures with computational effort that is comparable to that of the ALDA and AGGA. The final point of the study-with probably the greatest impact in the long run-is the efficiency of the combination of TP TDDFT, LCAO, and the PAW method. The method is not limited to pure Ag nanostructures. Our preliminary results show that it is also applicable to intermetallic nanostructures, such as Au-Ag core-shell NPs, as well as to nanostructures with molecular parts, e.g., ligandprotected AuNPs [53] and metallic nanoantennas connected by molecular tunnel junctions [2].

Altogether the combination of the adiabatic GLLB-SC, the LCAO-PAW with extended basis, and the time-propagation methods allows for accurate simulations of LSPRs in noblemetal nanostructures towards macroscopic sizes.

\section{ACKNOWLEDGMENTS}

We thank the Academy of Finland for financial support through Projects No. 269402 and No. 273499, through its Centres of Excellence Programme under Project No. 251748, 
and through its National Graduate School of Materials Physics. T.P.R. acknowledges financial support from the Vilho, Yrjö and Kalle Väisälä Foundation. We thank CSC - IT Center for Science Ltd. (Espoo, Finland) and the Aalto Science-IT project for computational resources. A.H.L. acknowledges support from the European Research Council Advanced Grant DYNamo (Proposal No. 267374) and Grupos Consolidados UPV/EHU del Gobierno Vasco (Grant No. IT-578-13).
[1] T. R. Jensen, M. D. Malinsky, C. L. Haynes, and R. P. Van Duyne, J. Phys. Chem. B 104, 10549 (2000).

[2] S. F. Tan, L. Wu, J. K. W. Yang, P. Bai, M. Bosman, and C. A. Nijhuis, Science 343, 1496 (2014).

[3] J. M. Bingham, J. N. Anker, L. E. Kreno, and R. P. Van Duyne, J. Am. Chem. Soc. 132, 17358 (2010).

[4] K. M. Mayer and J. H. Hafner, Chem. Rev. 111, 3828 (2011).

[5] K. A. Willets and R. P. Van Duyne, Annu. Rev. Phys. Chem. 58, 267 (2007)

[6] M. Xiao, R. Jiang, F. Wang, C. Fang, J. Wang, and J. C. Yub, J. Mater. Chem. A 1, 5790 (2013).

[7] S. Pillai and M. Green, Sol. Energy Mater. Sol. Cells 94, 1481 (2010).

[8] V. Giannini, A. I. Fernández-Domínguez, S. C. Heck, and S. A. Maier, Chem. Rev. 111, 3888 (2011).

[9] K. L. Kelly, E. Coronado, L. L. Zhao, and G. C. Schatz, J. Phys. Chem. B 107, 668 (2003).

[10] C. Noguez, J. Phys. Chem. C 111, 3806 (2007).

[11] K.-P. Charlé, L. König, S. Nepijko, I. Rabin, and W. Schulze, Cryst. Res. Technol. 33, 1085 (1998).

[12] J.-O. Joswig, L. O. Tunturivuori, and R. M. Nieminen, J. Chem. Phys. 128, 014707 (2008).

[13] C. Xia, C. Yin, and V. V. Kresin, Phys. Rev. Lett. 102, 156802 (2009).

[14] X. López-Lozano, C. Mottet, and H.-C. Weissker, J. Phys. Chem. C 117, 3062 (2013).

[15] E. Prodan and P. Nordlander, Chem. Phys. Lett. 352, 140 (2002).

[16] L. Serra and A. Rubio, Phys. Rev. Lett. 78, 1428 (1997).

[17] E. Runge and E. K. U. Gross, Phys. Rev. Lett. 52, 997 (1984).

[18] P. Hohenberg and W. Kohn, Phys. Rev. 136, B864 (1964).

[19] W. Kohn and L. J. Sham, Phys. Rev. 140, A1133 (1965).

[20] G. Barcaro, L. Sementa, A. Fortunelli, and M. Stener, J. Phys. Chem. C 118, 12450 (2014).

[21] J. Yan, K. W. Jacobsen, and K. S. Thygesen, Phys. Rev. B 84, 235430 (2011).

[22] C. Sönnichsen, T. Franzl, T. Wilk, G. von Plessen, J. Feldmann, O. Wilson, and P. Mulvaney, Phys. Rev. Lett. 88, 077402 (2002).

[23] X. López-Lozano, H. Barron, C. Mottet, and H.-C. Weissker, Phys. Chem. Chem. Phys. 16, 1820 (2014).

[24] J. A. Scholl, A. L. Koh, and J. A. Dionne, Nature (London) 483, 421 (2012)

[25] J. Tiggesbäumker, L. Köller, K.-H. Meiwes-Broer, and A. Liebsch, Phys. Rev. A 48, R1749(R) (1993).

[26] H. Haberland, Nature (London) 494, E1 (2013).

[27] M. Kuisma, J. Ojanen, J. Enkovaara, and T. T. Rantala, Phys. Rev. B 82, 115106 (2010).

[28] O. Gritsenko, R. van Leeuwen, E. van Lenthe, and E. J. Baerends, Phys. Rev. A 51, 1944 (1995).
[29] J. Yan, K. W. Jacobsen, and K. S. Thygesen, Phys. Rev. B 86, 241404 (2012).

[30] J. P. Perdew, A. Ruzsinszky, G. I. Csonka, O. A. Vydrov, G. E. Scuseria, L. A. Constantin, X. Zhou, and K. Burke, Phys. Rev. Lett. 100, 136406 (2008).

[31] O. V. Gritsenko, R. V. Leeuwen, and E. J. Baerends, Int. J. Quantum Chem. 61, 231 (1997).

[32] M. Städele, J. A. Majewski, P. Vogl, and A. Görling, Phys. Rev. Lett. 79, 2089 (1997).

[33] I. E. Castelli, T. Olsen, S. Datta, D. D. Landis, S. Dahl, K. S. Thygesen, and K. W. Jacobsen, Energy Environ. Sci. 5, 5814 (2012).

[34] J. B. Krieger, Y. Li, and G. J. Iafrate, Phys. Rev. A 45, 101 (1992).

[35] D. Pines and D. Bohm, Phys. Rev. 85, 338 (1952).

[36] P. E. Blöchl, Phys. Rev. B 50, 17953 (1994).

[37] J. Enkovaara, C. Rostgaard, J. J. Mortensen, J. Chen, M. Dułak, L. Ferrighi, J. Gavnholt, C. Glinsvad, V. Haikola, H. A. Hansen, H. H. Kristoffersen, M. Kuisma, A. H. Larsen, L. Lehtovaara, M. Ljungberg, O. Lopez-Acevedo, P. G. Moses, J. Ojanen, T. Olsen, V. Petzold, N. A. Romero, J. Stausholm-Møller, M. Strange, G. A. Tritsaris, M. Vanin, M. Walter, B. Hammer, H. Häkkinen, G. K. H. Madsen, R. M. Nieminen, J. K. Nørskov, M. Puska, T. T. Rantala, J. Schiøtz, K. S. Thygesen, and K. W. Jacobsen, J. Phys.: Condens. Matter 22, 253202 (2010).

[38] A. H. Larsen, M. Vanin, J. J. Mortensen, K. S. Thygesen, and K. W. Jacobsen, Phys. Rev. B 80, 195112 (2009).

[39] A. H. Larsen, J. Kleis, K. S. Thygesen, J. K. Nørskov, and K. W. Jacobsen, Phys. Rev. B 84, 245429 (2011).

[40] K. Yabana and G. F. Bertsch, Phys. Rev. B 54, 4484 (1996)

[41] M. Walter, H. Häkkinen, L. Lehtovaara, M. Puska, J. Enkovaara, C. Rostgaard, and J. J. Mortensen, J. Chem. Phys. 128, 244101 (2008).

[42] M. E. Casida, in Recent Advances in Density Functional Methods, Part I, edited by D. P. Chong (World Scientific, Singapore, 1995), p. 155.

[43] K. Yabana and G. F. Bertsch, Phys. Rev. A 60, 3809 (1999).

[44] L. S. Blackford, J. Choi, A. Cleary, E. D’Azevedo, J. Demmel, I. Dhillon, J. Dongarra, S. Hammarling, G. Henry, A. Petitet, K. Stanley, D. Walker, and R. C. Whaley, SCALAPACK Users' Guide (Society for Industrial and Applied Mathematics, Philadelphia, PA, 1997).

[45] J. Dongarra and R. C. Whaley, LAPACK working note 94, Technical Report No. UT-CS-95-281, (1997).

[46] D. Schooss, M. Blom, J. Parks, B. von Issendorff, H. Haberland, and M. Kappes, Nano Lett. 5, 1972 (2005).

[47] F. Baletto, R. Ferrando, A. Fortunelli, F. Montalenti, and C. Mottet, J. Chem. Phys. 116, 3856 (2002).

[48] P. Johnson and R. Christy, Phys. Rev. B 6, 4370 (1972).

[49] A. Coomar, C. Arntsen, K. A. Lopata, S. Pistinner, and D. Neuhauser, J. Chem. Phys. 135, 084121 (2011). 
[50] A. Sakko, T. P. Rossi, and R. M. Nieminen, J. Phys.: Condens. Matter 26, 315013 (2014).

[51] J. P. Perdew, K. Burke, and M. Ernzerhof, Phys. Rev. Lett. 77, 3865 (1996).

[52] S. Bernadotte, F. Evers, and C. R. Jacob, The Journal of Physical Chemistry C 117, 1863 (2013).

[53] S. Malola, L. Lehtovaara, J. Enkovaara, and H. Häkkinen, ACS Nano 7, 10263 (2013).
[54] H. Häkkinen, M. Moseler, O. Kostko, N. Morgner, M. A. Hoffmann, and B. von Issendorff, Phys. Rev. Lett. 93, 093401 (2004).

[55] B. Wortmann, K. Mende, S. Duffe, N. Grönhagen, B. von Issendorff, and H. Hövel, Phys. Status Solidi B 247, 1116 (2010).

[56] T. P. Rossi, S. Lehtola, A. Sakko, M. J. Puska, and R. M. Nieminen, J. Chem. Phys. 142, 094114 (2015).

[57] A. Castro, A. Rubio, and M. J. Stott, Can. J. Phys. 81, 1151 (2003). 